\title{
Seasonal Body Composition Variation Amongst Elite European Professional Soccer Players: An Approach of Talent Identification
}

\author{
by \\ Adam Lee Owen 1,2, Carlos Lago-Peñas 3,4, Gordon Dunlop ${ }^{1}$, Rouissi Mehdi ${ }^{5}$, \\ Moktar Chtara ${ }^{5}$, Alexandre Dellal 2,6
}

The primary aim of the investigation was to study the seasonal changes in body composition in elite European soccer players and identify key playing positional differences. Twenty-two players (age $=24 \pm 3.7$ years, body height $=$ $180.45 \pm 5.12 \mathrm{~cm}$, body mass $=76.66 \pm 5.34 \mathrm{~kg}$ ) were tested. Players' mass $(\mathrm{kg})$, lean body mass $(\mathrm{LBM})$, fat free mass (FFM), fat mass (FM), muscle girths $(M G)$ and sum of 8 skinfolds $(\mathrm{mm})$ were measured across 5 time points $(\mathrm{T0}=$ Start of pre-season training; $T 1=$ End of pre-season training; $T 2=$ Mid-season; $T 3=$ End of mid-season break; $T 4=$ End of season). Players were divided into their tactical positional roles for analysis. The specific positions they were divided into included defenders $(n=8)$, midfielders $(n=8)$ and forwards $(n=6)$. Assessment of training and matchplay exposure were also recorded. Sites-4, Sites-7, Sites-8 and Fat Mass decreased dramatically from T0 to T1 and T2 in all playing positions $(p<0.01)$, while no remarkable differences were found between T2, T3 and T4. Except for defenders, calf girth and lean mass were higher in T2, T3 and T4 compared to T0 and T1 $(p<0.01)$. Midfielders were found to be leaner than forwards and defenders in all data collection time point sessions. Defenders showed higher values in calf girth and lean body mass than midfielders and forwards. It can be concluded from this investigation that there are large variances $n$ positional body composition profiles amongst professional European soccer players. Furthermore, significant changes are prevalent and occur across the season from LBM, FFM, MG and skinfold assessment amongst European elite level soccer players.

Key words: human performance, positional analysis, anthropometry.

\section{Introduction}

Although a high proportion of soccer specific training content is performed collectively as a team, it is crucial that the positional demands of the game and subsequent training approaches are taken into consideration to maximize performance. Soccer as a sport at the elite level is suggested to require many physical, physiological and psychological characteristics to align in order to become successful (Owen et al., 2015). Previous research has suggested that body composition analyses are vital when determining success in elite sport (Casajús, 2011; Clark et al., 2008). Casajús (2011) recently revealed notable fat mass reductions in line with an increase in fat free mass without hardly any variation in body mass between in-season testing sessions. However,

\footnotetext{
1 - BenficaLAB, Sport Lisboa e Benfica Sport Science Department, Lisbon, Portugal.

2 - Centre de Recherche et d'Innovation sur le Sport, Université Claude Bernard Lyon.1, Lyon, France.

3 - Faculty of Educational Sciences and Sport, University de Vigo, Pontevedra, Spai.

4 - Department of Sport \& Exercise sciences, Soccer Research Group, University of Sunderland, Sunderland, UK.

5 - Tunisian Research Laboratory, National Centre of Medicine E Science in Sport, Tunis, Tunisia.

6 - Santy Orthopedicae Clinical Centre, FIFA Medical Centre of Excellence, Lyon, France.
} 
contrasting findings were described by Clark et al. (2008) who found no notable changes in body composition across the season.

Variables discussed and highlighted among the literature are suggested to be largely dependent on heredity while also being correlated with age, gender, dietary habits, training and competitive match play loadings. The induced physical stress of professional soccer training and competitive matches across a whole season may modulate players' body composition and plays a major role in their physical profile (Ekblom, 1986); however, body composition changes in professional soccer during the season have only been scarcely investigated (Casajús, 2011; Clark et al., 2008; Lago-Peñas et al., 2013). Positional differences are commonly cited within the literature when detailing the activity profiles and workloads of soccer players. Literature has highlighted the positional differences in player time and motion analysis profiles within professional soccer (Bloomfield et al., 2007; Di Salvo et al., 2007), which subsequently impose significant variations of the physical expenditure of players in both competition and training (Bloomfield et al., 2007; Dellal et al., 2012). Bangsbo (1994) described how midfield players tended to undertake more periods of low to moderate intensity activity for longer duration, while being stationary for significantly less time when compared to other outfield positional roles. This in turn corresponded to more total distance covered during competitive matches compared to players in a more defensive or attacking role (Castellano et al., 2012).

Furthermore, attacking based players (centre and wide forwards) were found to perform more maximal and longer duration sprints when compared to midfielders (Castellano et al., 2012; Di Salvo et al., 2007). Different soccer related activities such as jumping, long passing, tracking opposition runs, and the positional differences within the game place a different demand and intensity on the body (Bloomfield et al., 2007). Based on this changed response of intensity level as a direct response to positional demands, the energetic costs placed on players may also significantly affect body compositional data across the season.

Despite concerns about the fact that body composition variables are an essential part of the evaluation and selection process of elite level athletes, standard data on such variables within elite level European soccer are still very limited. As a result, the purpose of this particular study was to highlight the changes and differences in relation to fat displacement and playing positions amongst professional players in addition to highlighting other body compositional changes across a competitive season.

It may be hypothesized that variations in soccer player's body composition occur gradually across the season. Additionally, the positional role played within training and matches may influence changes in body composition variables tested based on the various training and match based workloads and variance in energy cost.

\section{Methods}

\section{Participants}

Twenty-two elite male European soccer players were tested and assessed throughout this investigation (age $=24 \pm 3.7$ years, mean height $=$ $180.45 \pm 5.12 \mathrm{~cm}$, mean body mass $=76.66 \pm 5.34$ $\mathrm{kg})$. The players' mass $(\mathrm{kg})$, lean body mass (LBM), fat free mass (FFM), fat mass (FM), muscle girths (MG) and sum of 4, 7 and 8 skinfolds (mm) for the determination of fat displacement were measured across 5 time points of the competitive season $(\mathrm{T} 0=$ Start of pre-season training; $\mathrm{T} 1=$ End of pre-season training; T2 = Mid-season; T3 = End of mid-season break; T4 = End of season). Players were divided into their tactical units for analysis (Defenders $\mathrm{n}=8$, Midfielders $\mathrm{n}=8$ and Forwards $\mathrm{n}=6$ ). Written informed consent was received from each subject following the explanation of the aims and potential benefits and risks associated to participation in the investigation. Procedures for the study were in accordance with the Declaration of Helsinki and approved by the ethical committee of the University and by the Servette Centre for Football Research Group respectively, before assessments were commenced.

\section{Design and Procedures}

To examine seasonal changes in body composition across various playing positions amongst elite European professional soccer players, data was collected across a 46-week period, encompassing the complete schedule of the 2014-2015 competitive season. To correspond with the periodised training structure implemented, body composition analysis was 
performed across 4 phases of training, initiating in June (pre-season) and concluding in May (end of season) and was inclusive of 5 specific data collection time points as shown in Figure 1.

Body compositional assessment was supervised and performed by the sport science and medical department of the club with testing performed by the same ISAK certified practitioner throughout the seasonal investigation period in accordance with the standardised techniques adopted by the International Society for the Advancement of Kinanthropometry (ISAK). Players were instructed to arrive at the laboratory in a post-absorptive (overnight fast), hydrated state prior to assessing body composition.

In total, fourteen assessment variables were examined across the investigation period as part of the restricted body compositional profile protocol implemented. Skinfold thickness (mm) was measured at 8 identified anatomical landmark sites, inclusive of subscapular, bicep, tricep, iliac crest, supraspinale, abdominal, front thigh and medial calf using a Harpenden caliper (British Indicators Ltd., St. Albans, UK). To complete the restricted body composition profile, basic measurements of stature (stadiometer) and body mass (CIRCA, California, USA) were collected in addition to five girths landmark sites. Using a specific anthropometric tape (Gulick, Creative Health Products, Plymouth, MI) measurement of the arm (at the level of the midacromialeradiale site) was performed for both relaxed and arm flexed / tensed positions, while waist (minimum), gluteal and calf (maximum) girths were also documented.

All measurements were taken in accordance with the guidelines of the International Society for the Advancement of Kinanthropometry (ISAK). All landmark sites were firstly identified before any measurements were recorded, with each site being measured in succession to avoid experimenter bias and reduce the effects of skinfold-compressibility prior to repeating measurements for a second time. A further third measurement was made in instances where the technical error of measurement (TEM) was not within $5 \%$ of the first skinfold measurement recorded, or within $1 \%$ of the first for all other dimensions, respectively. Previous test-retest reliability scores for the sum of 4, 7 and 8 skinfolds and body measures within similar populations had been high (ICCs range 0.92 to 0.97) (Lago-Peñas et al., 2013). With respect to data analysis, on occasions where three measures were required, the median value, rather than the mean value of the data recorded was used for analysis.

\section{Statistical analysis}

Means \pm standard deviations [SD] were used to describe all the different playing positional body composition variables throughout the investigation. The normality of data was verified using the Kolmogorov-Smirnov test. Friedman's two-way ANOVA for repeated measures was used to determine the differences between the phases of the study, with each subject serving as his own control. When a significant $\chi^{2}$ value was achieved, appropriate Wilcoxon posthoc test procedures were used to locate the difference between $\mathrm{T} 0$ and T1-T4. The effect size was calculated for all ANOVAs using a partial eta-squared. Values of $0.01,0.06$ and above 0.15 were considered as small, medium and large, respectively (Cohen, 1988). Statistical analyses were performed using SPSS software statistical package (SPSS Inc., Chicago, IL, version. 16.0), and statistical significance was set at $p<0.05$.

\section{Results}

\section{Body composition according to the positional role}

The mean values ( \pm SD) of body composition (calf girth, Sites-4, Sites-7, Sites-8, Lean mass, and Fat mass) distributed across five separate testing points, and organised within playing positions within the season are presented in Table 1.

Friedman's two-way ANOVA for repeated measures demonstrated a significant difference in all estimated body composition variables at the five data collection time points sessions $(p<0.05$ to 0.001$)$. Sites -4 , Sites-7, Sites- 8 and Fat Mass decreased dramatically from the start (T0) to the end of the pre-season (T1) and the Mid-season (T2) in all playing positions as highlighted in Figure 2. Sites-4, Sites-7, Sites- 8 and Fat Mass values were lower in T2, T3 and T4 compared to $\mathrm{T} 1$ and especially T0. However, as shown in Figure 1, no remarkable differences were found between $\mathrm{T} 2, \mathrm{~T} 3$ and $\mathrm{T} 4$. On the contrary, lean mass was significantly higher in T1 compared to $\mathrm{T} 0$ in all playing positions. Except for 
Defenders, calf girth increased from T0 to T1 $(p<$ 0.01 ). Calf girth and lean mass were higher in $\mathrm{T} 2$, $\mathrm{T} 3$ and T4 compared to T0 and T1. Values in T4 were slightly lower than in $\mathrm{T} 2$ and $\mathrm{T} 3$.

Midfielders had less fat mass than all the other playing positions. Additionally, midfielders were found to be leaner than forwards and defenders in all data collection time point sessions. Defenders showed higher values in calf girth and lean mass than midfielders and forwards. Sites-4, Sites-7, Sites- 8 and fat mass decreased in all playing positions from $\mathrm{T} 0$ to $\mathrm{T} 4$, however, values at the end of the season were slightly higher than in T2 and T3. Calf girth and lean mass increased from T0 to T4. However, values at the end of the season were higher than in $\mathrm{T} 2$ and $\mathrm{T} 3$.

\section{Discussion}

The study of body composition is important in those sports where body weight must be moved repeatedly against gravity. Body composition among soccer players is likely to change during the course of the competitive season as a result of training and competition, habitual activity, and diet. Similar to other investigations, the present study demonstrated significant intra-season differences in body composition. Fat mass decreased significantly at T1, T2, T3 and T4 compared with start of pre-season training (T0) which is in line with previous studies (Lago-Peñas et al., 2013). In the current study, the loss in body fat during the preseason period may have been a direct result of the high levels of aerobic, anaerobic, and muscle strength training performed during this period coupled with preseason training games on the back of a training cessation period. This is supported by Reilly (1996) who states that low body fat is a direct reflection of high training intensities, whereas Bangsbo (1994) states that match play places high demands on the aerobic energy system $(90 \%)$ and causes large energy expenditure; therefore, the body uses fat stores as energy. The players' percent body fat continued to decrease significantly at T2, T3 and T4 compared with T1. These results are similar to those provided by Lago-Peñas et al. (2013) who also studied professional soccer players and proposed that it may be as a result of regular training and match play. In addition, and as a result of the training influence on body composition, a significant increase in lean mass and calf girth in T1, T2, T3 and $\mathrm{T} 4$ compared with $\mathrm{T} 0$ was observed.

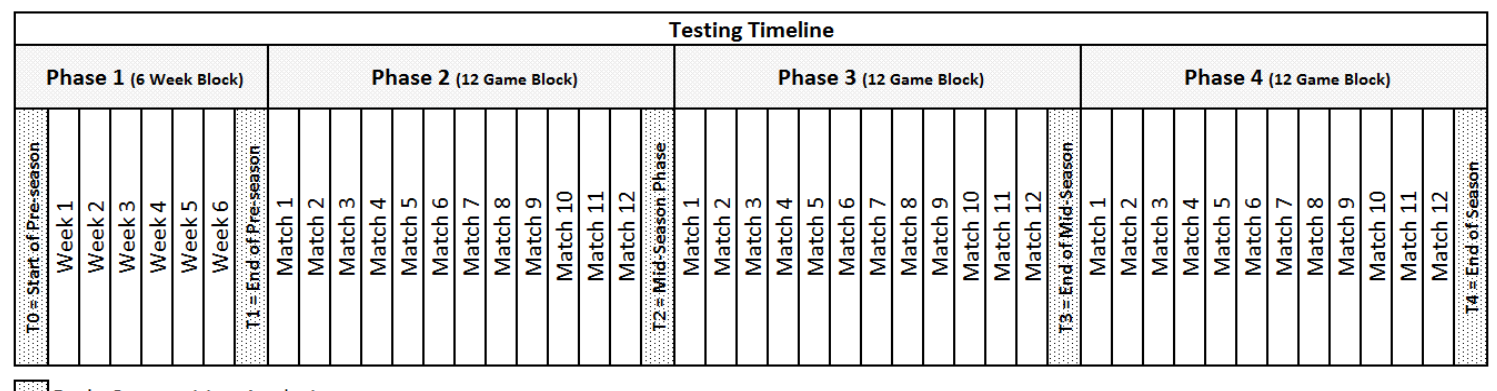

Body Composition Analysis

Figure 1

Body Composition Testing Timeline 
Table 1

Body composition in professional soccer players according to three playing positions at five different points across the season (mean $\pm S D, n=17$ ).

\begin{tabular}{|c|c|c|c|c|c|c|}
\hline & T0 & T1 & $\mathrm{T} 2$ & T3 & $\mathrm{T} 4$ & Chi-square \\
\hline \multicolumn{7}{|l|}{ Calf girth } \\
\hline All players & $37.15 \pm 2.14$ & $37.64 \pm 2.24$ & $37.81 \pm 2.17$ & $37.69 \pm 2.15$ & $37.44 \pm 2.07$ & $18.28^{* *}$ \\
\hline Defenders & $38.58 \pm 1.53$ & $38.85 \pm 1.77$ & $38.87 \pm 1.66$ & $38.85 \pm 1.71$ & $38.45 \pm 1.45$ & 6.23 \\
\hline Midfielders & $36.17 \pm 2.54$ & $36.83 \pm 2.78$ & $36.65 \pm 2.83$ & $36.32 \pm 2.58$ & $36.08 \pm 2.51$ & $12.48^{* *}$ \\
\hline Forwards & $36.62 \pm 1.56$ & $37.16 \pm 1.72$ & $37.92 \pm 1.31$ & $37.94 \pm 1.30$ & $37.84 \pm 1.47$ & $12.93^{* *}$ \\
\hline \multicolumn{7}{|l|}{ Sites 4} \\
\hline All players & $33.51 \pm 6.57$ & $30.08 \pm 6.02$ & $27.21 \pm 4.30$ & $26.62 \pm 4.18$ & $28.08 \pm 4.60$ & $39.91^{* *}$ \\
\hline Defenders & $36.12 \pm 5.98$ & $31.20 \pm 4.16$ & $27.98 \pm 4.22$ & $27.27 \pm 4.19$ & $27.85 \pm 3.32$ & $16.00^{* *}$ \\
\hline Midfielders & $30.18 \pm 5.78$ & $27.85 \pm 5.79$ & $25.31 \pm 4.07$ & $25.22 \pm 3.43$ & $27.10 \pm 3.46$ & $18.00^{* *}$ \\
\hline Forwards & $34.36 \pm 7.65$ & $31.42 \pm 8.37$ & $28.58 \pm 4.75$ & $27.51 \pm 5.35$ & $29.54 \pm 7.20$ & $9.28^{*}$ \\
\hline \multicolumn{7}{|l|}{ Sites 7} \\
\hline All players & $50.35 \pm 8.44$ & $46.54 \pm 7.95$ & $44.44 \pm 6.06$ & $43.23 \pm 5.83$ & $45.21 \pm 6.46$ & $32.68^{* *}$ \\
\hline Defenders & $52.92 \pm 7.86$ & $48.58 \pm 6.91$ & $45.92 \pm 4.51$ & $44.33 \pm 4.18$ & $45.48 \pm 4.68$ & $14.35^{* *}$ \\
\hline Midfielders & $45.65 \pm 6.49$ & $41.37 \pm 5.96$ & $40.93 \pm 5.22$ & $39.98 \pm 4.05$ & $43.45 \pm 5.61$ & $13.07^{* *}$ \\
\hline Forwards & $52.90 \pm 10.20$ & $50.30 \pm 9.21$ & $46.89 \pm 7.63$ & $45.81 \pm 8.23$ & $46.98 \pm 9.58$ & $14.99^{* *}$ \\
\hline \multicolumn{7}{|l|}{ Sites 8} \\
\hline All players & $63.51 \pm 11.87$ & $57.06 \pm 10.61$ & $53.52 \pm 7.89$ & $51.99 \pm 7.29$ & $55.01 \pm 7.96$ & $42.09^{* *}$ \\
\hline Defenders & $67.45 \pm 11.01$ & $59.18 \pm 8.13$ & $55.25 \pm 6.20$ & $53.38 \pm 4.82$ & $55.22 \pm 4.36$ & $15.60^{* *}$ \\
\hline Midfielders & $57.18 \pm 10.54$ & $51.33 \pm 9.18$ & $49.42 \pm 7.58$ & $48.18 \pm 5.71$ & $52.53 \pm 7.19$ & $15.69^{* *}$ \\
\hline Forwards & $66.38 \pm 13.41$ & $61.3 \pm 13.52$ & $56.37 \pm 9.46$ & $54.87 \pm 10.38$ & $57.74 \pm 12.11$ & $14.24^{* *}$ \\
\hline \multicolumn{7}{|l|}{ Lean mass } \\
\hline All players & $67.21 \pm 5.31$ & $68.23 \pm 5.49$ & $69.01 \pm 5.11$ & $68.95 \pm 5.24$ & $68.71 \pm 5.13$ & $29.93^{* *}$ \\
\hline Defenders & $69.88 \pm 4.37$ & $70.84 \pm 4.96$ & $71.18 \pm 4.79$ & $71.18 \pm 4.79$ & $70.66 \pm 4.24$ & $9.73^{*}$ \\
\hline Midfielders & $64.16 \pm 6.61$ & $64.83 \pm 6.52$ & $65.94 \pm 6.37$ & $65.94 \pm 6.37$ & $65.81 \pm 6.78$ & $12.73^{* *}$ \\
\hline Forwards & $67.68 \pm 3.03$ & $69.18 \pm 2.85$ & $69.88 \pm 2.86$ & $69.88 \pm 2.86$ & $69.86 \pm 2.28$ & $11.68^{*}$ \\
\hline \multicolumn{7}{|l|}{ Fat mass } \\
\hline All players & $7.47 \pm 1.78$ & $6.79 \pm 1.54$ & $6.35 \pm 1.17$ & $6.24 \pm 1.16$ & $6.56 \pm 1.21$ & $30.83^{* *}$ \\
\hline Defenders & $8.26 \pm 1.54$ & $7.23 \pm 1.04$ & $6.72 \pm 0.97$ & $6.58 \pm 0.81$ & $6.74 \pm 0.62$ & $13.33^{* *}$ \\
\hline Midfielders & $6.30 \pm 1.34$ & $5.76 \pm 1.09$ & $5.58 \pm 0.83$ & $5.50 \pm 0.60$ & $5.96 \pm 0.83$ & $11.06^{*}$ \\
\hline Forwards & $7.94 \pm 2.04$ & $7.48 \pm 2.04$ & $6.83 \pm 1.43$ & $6.70 \pm 1.69$ & $7.06 \pm 1.90$ & $12.00^{*}$ \\
\hline
\end{tabular}

*Significant difference: $p<0.05 ;{ }^{* *}$ Significant difference: $p<0.01$ between sessions.

T0 = Start of pre-season training; $T 1=$ End of pre-season training:

T2 = Mid-season; T3 = End of mid-season; T4 = End of season . 
Calf Girth (cm)

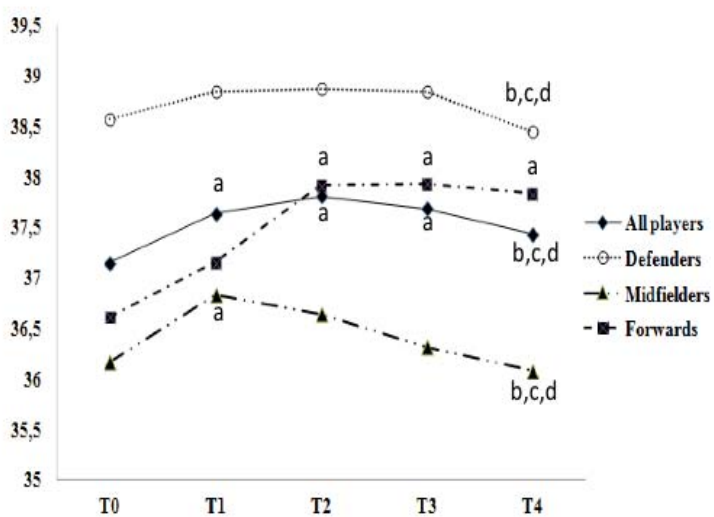

Sum of 7 Sites $(\mathrm{mm})$

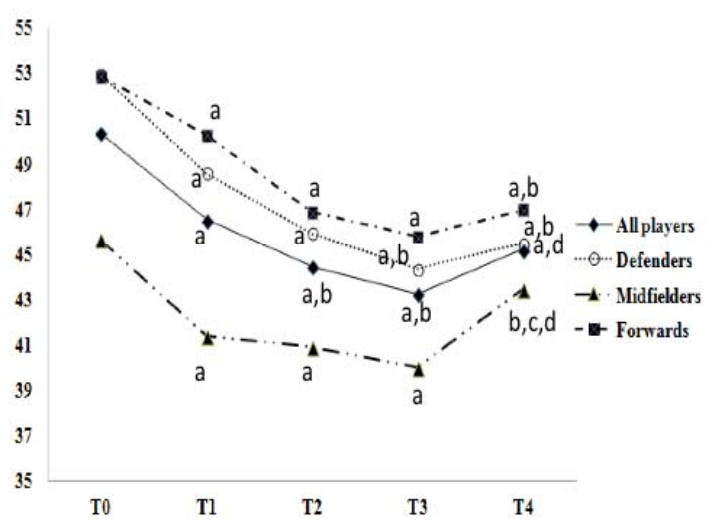

Lean Mass $(\mathrm{kg})$

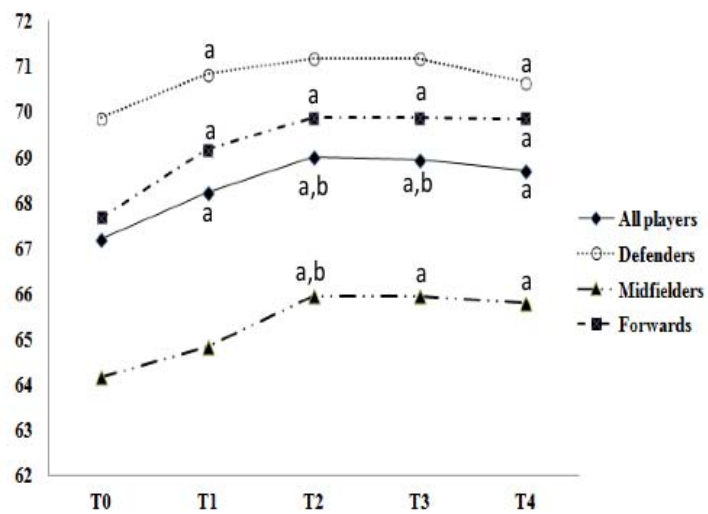

Sum of 4 Sites $(\mathrm{mm})$

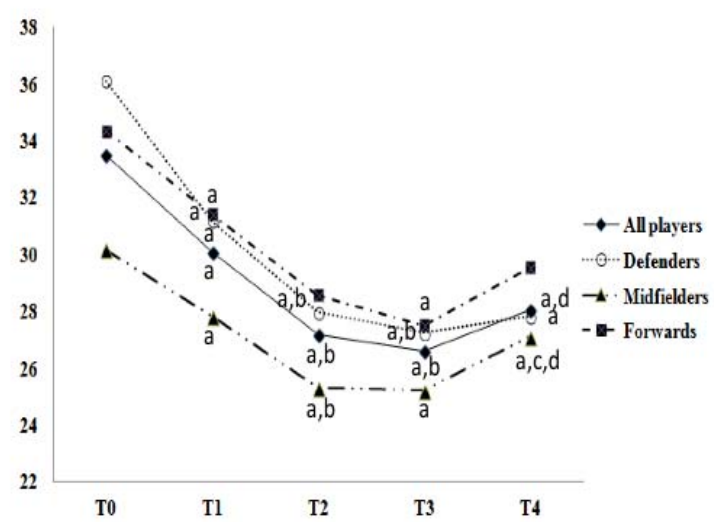

Sum of 8 Sites (mm)

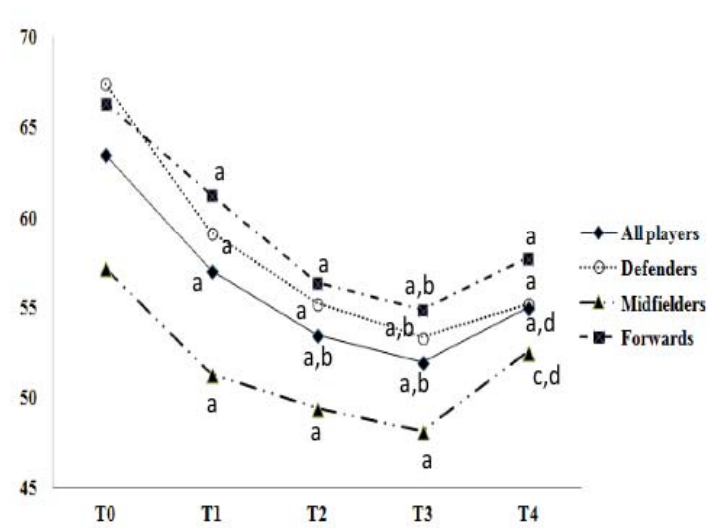

Fat mass (\%)

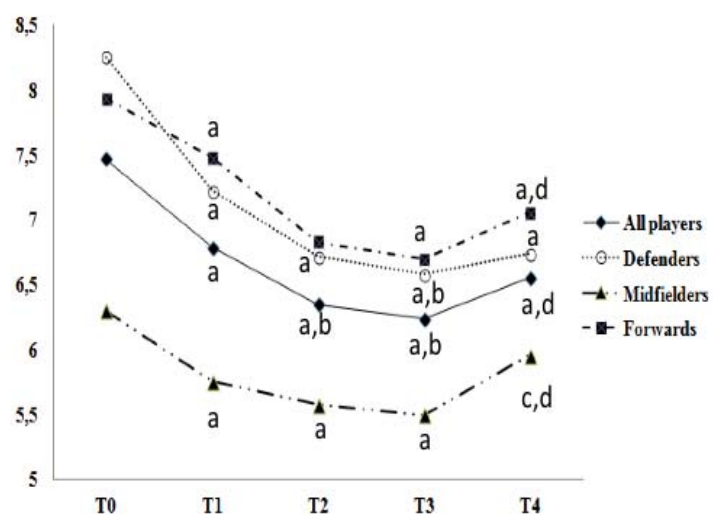

Figure 2

Seasonal changes in body composition variants.

a Significantly different from T0; ${ }^{b}$ Significantly different from

T1; c Significantly different from T2; ${ }^{d}$ Significantly different from T3 $(p<0.05)$. 
Elite level soccer players are usually exposed to demanding training schedules, which may include repeated, high-intensity sessions performed on consecutive days, multiple times per week. Each training session places high physical demands on players as they experience repeated moderate and rapid accelerations and decelerations, explosive jumps, and muscle eccentric loads (Di Salvo et al., 2007; Stolen et al., 2005). These training demands may impose strains to various physiological systems including musculoskeletal, nervous, immune and metabolic (Bangsbo, 1994). Thus, the knowledge on the relationship between playing exposure and physical fitness and body composition variables throughout the season would be helpful in the management of training programs.

Ensuring large subject participation within elite level soccer research is extremely difficult. For this particular investigation one of the limitations was that it did not include physical training and match outputs in terms of time motion analysis, energetic demands and other physical performance indicators shown through global positioning systems or a heart rate perspective. Possibility to include this data within the study would add significant value to the overall conclusions of the investigation. The variation of cultures across the current published literature in this area highlights a difficulty of being able to compare data across players. This may result from food types or lifestyle variation across these cultures having a different outcome when coupled with professional training environments (Casajús, 2011). Additionally, the country the experiment was conducted and its conditional environment also may be deemed as a limitation again based on the fact that it may be difficult to compare data across different continents. Therefore, with respect to this investigation, future research should look to draw from a larger subject pool in addition to increasing the number of testing sessions in order to add greater strength to the association of positional body site changes occurring and a period of the season. Future research would benefit from the development of a clear relationship between body compositional changes and internal and external physical output data across the season in addition to understanding the changes between players who are classed as starting players vs. substitutions.

In summary, this study provides normative data and performance standards for professional male soccer players, in order to allow individuals involved within the physical preparation or development to understand and determine the physical profile and body composition during the preseason and in-season phase. Furthermore, these specific profile standards may be used in a recruitment or talent selection process also to align specific physical qualities. Players who show adequate technical and tactical qualities may be further enhanced by bringing the physical qualities in line with values present in the current study as a benchmark for professional soccer players.

The results of this study suggest that soccer players appear to improve body composition during preseason. The "off season" appears to represent a period of significant reduction in sport-specific fitness in soccer players which in turn would reduce energy expenditure and cause a detraining effect and subsequent increased body-fat (Bloomfield et al., 2007). Offseason light "maintenance" training (2-3 times a week) should therefore be used to attempt to minimize these reductions. In doing so, players would already be closer to midseason peak match fitness at the start of the season, which would reduce the pressure on players to quickly regain large fitness deficits lost during the off-season. Also, fitness training elements of preseason training could be refined for quality, permitting more time for engaging in match play and thus enabling players to adapt to the apparent unique demands of actual game play earlier in the season. It is also recommended that to help maximize speed performance, players should carry as little body fat as possible.

It can be concluded from this investigation that there are large variances between positional body composition profiles amongst professional European soccer players. Furthermore, significant changes occur across the season from LBM, FFM, MG and skinfold assessment amongst elite European level soccer players. 


\section{Acknowledgements}

The authors would like to thank the soccer players for their participation and professionalism throughout the duration of the study; and the sport science \& medical staff. There are no funding sources and are no conflicts of interest surrounding this scientific investigation.

\section{References}

Bangsbo J. Energy demands in competitive soccer. J Sports Sci, 1994; 12: 5-12

Bloomield J, Polman R, O'Donoghue P. Physical demands of different positions in FA Premier League soccer. J Sports Sci Med, 2007; 6(1): 63-70

Casajus JA. Seasonal variation in fitness variables in professional soccer players. J Sports Med Phys Fit, 2001; 41(4): 463-469

Castellano J, Blanco-Villasenor A, Alvarez D. Contextual variables and time-motion analysis in soccer. Int J Sports Med, 2011; 32(6): 415-421

Clark NA, Edwards AM, Morton RH, Butterly RJ. Season-to-Season Variations of Physiological Fitness Within a Squad of Professional Male Soccer Players. J Sports Sci Med, 2008; 7(1): 157-165

Cohen J. Statistical power analysis for the behavioural sciences. L. Erbraum Associates, Hillside, NJ., 23-97; 1988

Dellal A, Drust B, Lago-Penas C. Variation of activity demands in small-sided soccer games. Int J Sports Med, 2012; 33(5): 370-375

Di Salvo V, Baron R, Tschan H, Calderon Montero FJ, Bachl N, Pigozzi F. Performance characteristics according to playing position in elite soccer. Int J Sports Med, 2007; 28(3): 222-227

Ekblom B. Applied physiology of soccer. Sports Med, 1986; 3(1): 50-60

Lago-Peñas C, Rey E, Lago-Ballesteros J, Dominguez E, Casais L. Seasonal variations in body composition and fitness parameters according to individual percentage of training completion in professional soccer players. Int SportMed J, 2013; 27(9): 2414-2419

Owen A, Dunlop G, Rouissi M, Chtara M, Paul D, Zouhal H, Wong DP. The relationship between lowerlimb strength and match-related muscle damage in elite level professional European soccer players. $J$ Sports Sci, 2015; 33(20): 2100-2105

Reilly T. Science and Soccer. London: E \& FN Spon; 1996

Stolen T, Chamari K, Castagna C, Wisloff U. Physiology of soccer: an update. Sports Med, 2005; 35(6): 501-536

\section{Corresponding author:}

\section{Dr. Adam Owen}

Sport Lisboa e Benfica Sport Science Department, Lisbon, Portugal.

BenficaLAB, Sport Lisboa e Benfica Sport Science Department, Lisbon, Portugal.

Phone: +41 799563991

Email: adamowen@outlook.com 